\title{
Intraoperative Frozen Section Assessment of Sentinel Lymph Node Biopsy in breast Carcinoma to Determine The Axillary Lymph Node Status.
}

\author{
Ramawatar R Soni ${ }^{1}$, Aishwarya Vinod Bhongade ${ }^{2 *}$, Anil T Deshmukh ${ }^{1}$, Rajendrasingh S Arora ${ }^{3}$, \\ Nafees Nomaan ${ }^{1}$ and Kirti S Dagdiya ${ }^{1}$ \\ ${ }^{1}$ Dept. of Pathology, Dr. P. D. M. Medical College, Amravati- Maharashtra, India \\ ${ }^{2}$ Dept. of Pathology, S.V.N. Govt Medical College, Yavatmal-445001, Maharashtra, India \\ ${ }^{3}$ Sujan surgical and cancer hospital and Amravati Cancer Foundation, Amravati, Maharashtra, India
}

\section{ABSTRACT}

Background: Breast cancer is commonest malignancy of females all over the world. Modified radical mastectomy is still considered as a standard for treating breast cancers. Axillary lymphadenectomy is achieved at the cost of significant morbidity, with a high acute complication rate and a high chronic lymphedema rate. Sentinel lymph node biopsy is associated with better arm function, better quality of life, a shorter hospital stay, and faster recommencement of daily activities than standard axillary lymph node dissection. There are inconsistencies and controversies regarding the pathological work-up of sentinel lymph nodes and no generally applied guidelines exist.

Methods: In total, 60 breast carcinoma cases were studied over a period of 2 years. The axillary lymph node status was determined intraoperatively in carcinoma breast by frozen section assessment of sentinel lymph node.

Results: Intraoperative frozen section had sensitivity of $90.3 \%$, specificity of $100 \%$, positive predictive value of $100 \%$, negative predictive value of $90.6 \%$ and diagnostic accuracy of $95 \%$. The sentinel lymph node biopsy had a false negative rate of $13.3 \% .16 .1 \%$ cases showed only sentinel lymph node positivity with non-sentinel lymph nodes free from metastasis. The positivity of sentinel lymph node for metastasis was dependent upon certain characteristics of patients such as tumour size, tumour location and grade of tumour and was independent of patient's age.

Conclusion: Intraoperative frozen section evaluation of sentinel lymph node (SLN) to predict the status of non-SLN can be recommended only in selected patients in early stage of disease.

\section{Keywords: Breast Cancer; Axillary Lymph Nodes; Sentinel Lymph Node; Intraoperative Frozen Section.}

\section{Introduction}

Breast cancer is commonest malignancy of females all over the world ${ }^{[1]}$ and second leading cause of death due to malignancy among females with an estimated 1.38 million new breast cancer cases diagnosed each year. ${ }^{[2]}$ In India, breast cancer incidence has overtaken cervical cancer incidence in most urban registries. ${ }^{[3]}$

Axillary lymph node dissection (ALND) was standard of care for a long time and considered necessary for locoregional control as well as for staging purposes. ${ }^{[4,5]}$ Axillary lymphadenectomy is achieved at the cost of significant morbidity, with a high acute complication rate and a high chronic lymphedema rate. ${ }^{[6-8]}$ Many authors found that sentinel lymph node biopsy was associated with better arm function, better quality of life, a shorter hospital stay, and faster recommencement of daily activities than standard axillary lymph node dissection. ${ }^{[4]}$ However, there are inconsistencies and controversies regarding the pathological work-up of SLNs and no generally applied guidelines exist. During sentinel lymph node biopsy (SLNB) harvested sentinel lymph nodes can be examined by frozen section analysis.

According to ESMO and ASCO guidelines, women without sentinel lymph node (SLN) metastases should not receive axillary lymph node dissection (ALND); women with one to two metastatic SLNs planning to undergo breast-conserving surgery with whole-breast radiotherapy should not undergo ALND (in most cases); women with SLN metastases who will undergo mastectomy should be offered ALND..$^{[9,10]}$ SLNB is not routinely recommended as replacement of formal axillary dissection, in view of higher degree of nodal positivity in India. ${ }^{[2]}$

The study was carried out to determine the sensitivity, specificity, positive predictive value, negative predictive value and diagnostic accuracy of frozen analysis of sentinel lymph node biopsy and to determine the significance of 
sentinel lymph node biopsy findings to predict the status of non-sentinel lymph nodes (non-SLNs) and to take appropriate decision/strategy regarding extent of surgery (cALND- completion Axillary Lymph Node Dissection) in future.

\section{Materials and Methods}

Total 60 patients were studied in the span of two years. Patients of all age groups undergoing surgery for breast cancer with unknown lymph node status were subjected to intraoperative frozen section assessment of sentinel lymph node with due informed consent. Patients with preoperative diagnosis of metastatic positive axillary lymph nodes were excluded. The study has been carried out after the approval of Institutional Ethics Committee.

Procedure: Preoperatively, after induction of general anesthesia, 3-5 $\mathrm{ml}$ of methylene blue dye was injected peritumourally. After breast mastectomy during axillary dissection, the SLNs (sentinel lymph nodes) were identified intra-operatively by visual inspection for blue dye. Only those nodes which were blue in colour, were labelled as the sentinel lymph nodes in the operation room and sent to the pathology laboratory immediately as separate specimen in plain container. While the nodal tissue was processed by frozen section the modified radical mastectomy surgery was continued.

All extracapsular fat was removed and the number of nodes was measured. Lymph nodes larger than $5 \mathrm{~mm}$ in diameter were bisected, whereas lymph nodes less than or equal to $5 \mathrm{~mm}$ in diameter were not bisected but completely submitted for frozen section analysis.

The frozen analysis was done using cryostat. Bisected or entire lymph nodes were kept on cryostat chuck and tissue freezing medium was applied over the chuck. Albumin was applied over slide and sections were taken on slide. The slides were stained with rapid haematoxylin and eosin stain. As soon as malignant cells were seen, this was reported to the operating surgeon.

However, complete axillary dissection of the patient was done irrespective of sentinel lymph node status. The remaining tissue from the frozen material was thawed, set in cassettes, fixed in buffered formalin and embedded in paraffin and further routine histopathological procedure was followed. The subsequent operative specimen was subjected to routine histopathological examination.

The results were tabulated stressing on following points:age of patient, tumour size, tumour location, number of SLNs obtained, average diameter of SLN, diagnosis of SLN on frozen section, diagnosis of SLN on subsequent H\&E staining, diagnosis of other axillary lymph nodes
(non-SLNs), diagnosis of the operated specimen. Diagnosis of SLN and non-SLN was given as positive or negative for metastasis. The Statistical software namely SPSS 16.0 and OpenEpi were used for the analysis of the data. Statistical analysis was done using mean and Chi-square test.

\section{Result}

Table 1 shows positive and negative cases on frozen section vs. positive and negative cases on paraffin section. From the table, true positive (TP) are the cases which showed metastasis both on frozen section as well as paraffin section i.e. 28. True negative $(\mathrm{TN})$ are the cases which did not showed any metastasis both on frozen section and paraffin section i.e. 29. False negative (FN) are the cases which did not showed metastasis on frozen section but were actually positive for metastasis on paraffin section i.e. 3 . False positive (FP) are the cases which showed metastasis on frozen section but did not actually had metastasis on paraffin section. There was no false positive case. With the help of these values, sensitivity $90.3 \%$, specificity $100 \%$, positive predictive value (PPV) $100 \%$, negative predictive value (NPV) $90.6 \%$ and diagnostic accuracy of frozen section $95 \%$ were calculated.

Table 2 shows positive and negative cases of SLN vs. positive and negative cases of non-SLN. From this, the false negative rate of SLN to detect the axillary lymph node status was $13.3 \%$ i.e. 4 cases were negative for metastasis on frozen section whereas the non-SLN showed metastasis. Also, $16.1 \%$ i.e. 5 cases showed metastasis in SLN whereas the non-SLNs of these cases did not showed any metastasis.

Out of 60 cases studied 28 cases showed SLN positive for metastasis. Out of these 28 cases maximum cases (11 cases) were in age group 41-50 years. However $p$ value found out to be $>0.05$ (not significant), there is no association of age with SLN positivity. It was observed that as the size of tumour increased, metastasis was observed in SLN. Out of 60 cases, maximum number of cases (24) had tumour located in upper outer quadrant. Out of these 24 cases, 15 cases $(62.5 \%)$ showed SLN positivity. However, this is statistically not significant ( $\mathrm{p}>0.05)$. Out of 60 cases, 54 were diagnosed as invasive ductal carcinoma (IDC). Out of 13 cases diagnosed as IDC grade III, 11 cases (84.6\%) showed SLN metastasis. This is statistically significant $(\mathrm{p}<0.05)$.

\section{Discussion}

In the present study the sensitivity, specificity, PPV, NPV and false negative rate was observed as $90.3 \%, 100 \%$, $100 \%, 90.6 \%$ and $13.3 \%$ respectively. This is comparable with previous studies of Mitchell M 2004 ${ }^{[11]}$, Gipponi M 
Table 1: POSITIVE AND NEGATIVE SLN (SENTINEL LYMPH NODES) ON FROZEN SECTION VS. PARAFFIN SECTION (ROUTINE H\&E).

\begin{tabular}{|l|c|c|c|c|}
\hline & \multicolumn{3}{|c|}{ On paraffin section } & Metastasis - \\
\hline On frozen section & & Metastasis + & 0 & 28 \\
\hline & Metastasis + & 28 & 29 & 32 \\
\hline & Metastasis - & 03 & $\mathbf{2 9}$ & 6 \\
\hline
\end{tabular}

Table 2: POSITIVE AND NEGATIVE FOR METASTASIS IN SLN VS. NON- SLN.

\begin{tabular}{|l|c|c|c|c|}
\hline & \multicolumn{3}{|c|}{ Metastasis in Non- SLN } & Total \\
\hline Metastasis in SLN & & Present & 05 & 31 \\
\hline & Present & 26 & 25 & 29 \\
\hline Total & Absent & 04 & 30 & 60 \\
\hline
\end{tabular}

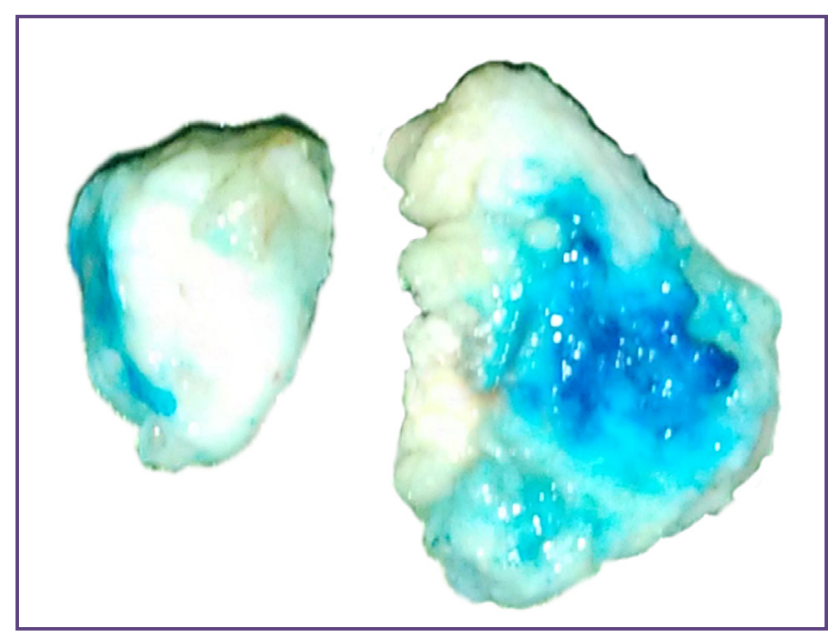

Photograph 1 : Gross photograph of SLN.

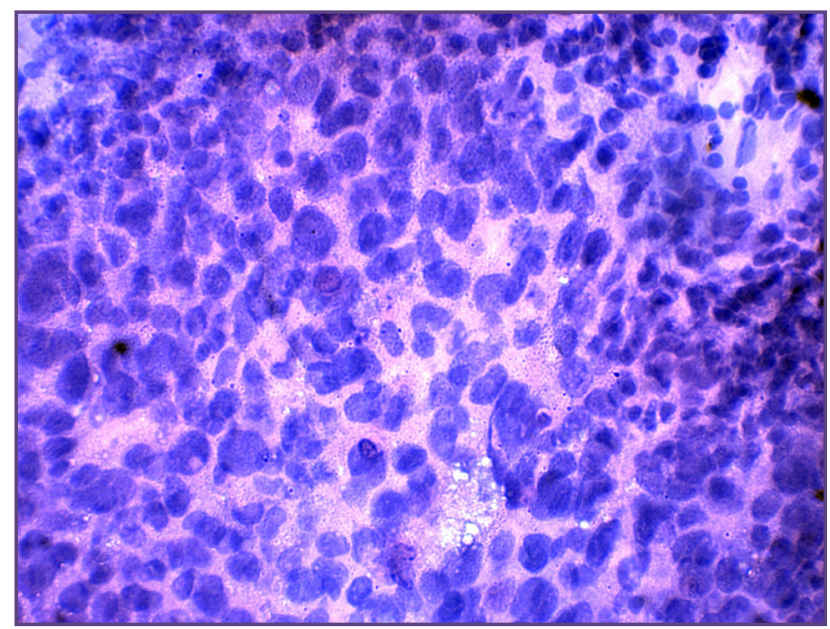

Photomicrograph 3: Frozen section of positive SLN ( rapid H\&E, 40X)

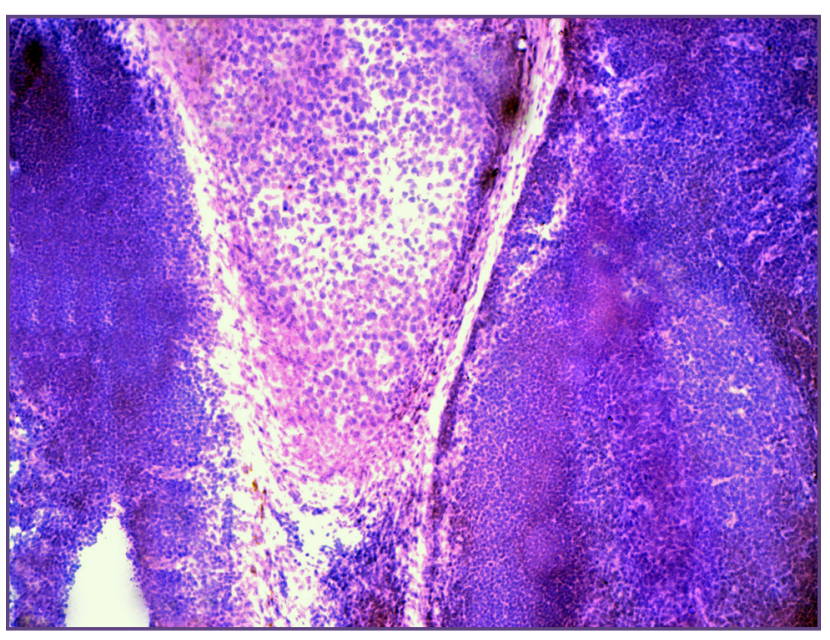

Photomicrograph 2: Frozen section of positive SLN (rapid H\&E, 10X).

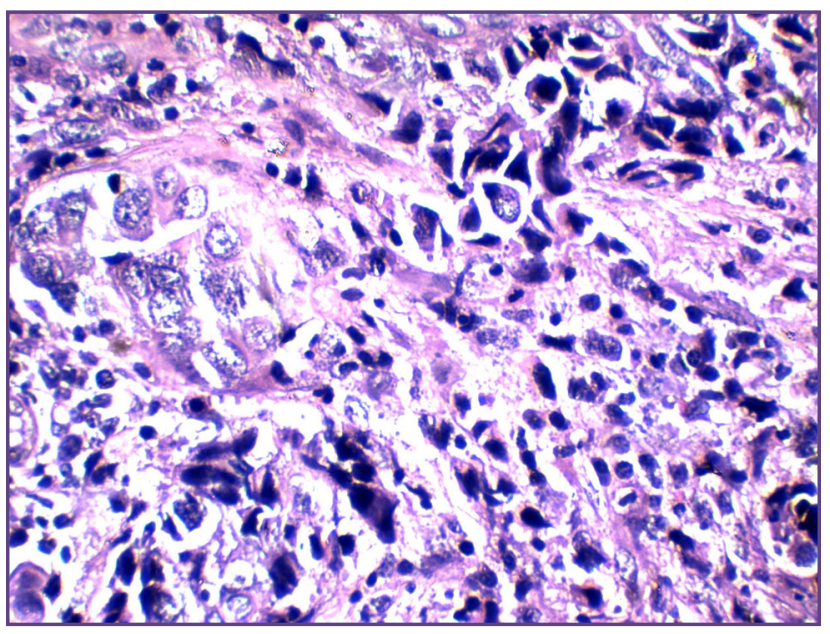

Photomicrograph 4: Paraffin section of positive SLN ( H\&E, 40X) 
et al 2004 $4^{[12]}$ and Tille J C et al 2009 ${ }^{[13]}$. Mitchell M 2004 predicted the axillary lymph node status with a sensitivity of $83 \%$, specificity of $100 \%$, accuracy of $96 \%$ and false negative rate of $13.3 \%$ of frozen section assessment of SLN. Gipponi M et al 2004 observed the frozen section assessment to have sensitivity of $93-95 \%$, specificity of $100 \%$, PPV of $97-98 \%$, NPV of $95-97 \%$ and false negative rate of $8.6 \%$. Tille $\mathrm{JC}$ et al 2009 found out the sensitivity of frozen section assessment as $83.3 \%$, specificity as $100 \%$, PPV as $100 \%$, NPV as $91.9 \%$ and false negative rate as $16.7 \%$.

The three cases wherein SLNs were negative for metastasis on frozen section and positive in paraffin section were reviewed again. In one case, the metastatic cells were small cells and so uniformly distributed that it might have been confused with lymphocytes. In other two cases, there was a small subscapular area showing metastatic cells in paraffin section which might have not been included in the section taken for frozen analysis.

In present study there were $16.1 \%$ cases which showed metastasis exclusively in SLN and in non-SLNs no malignant cells were seen. The present study is comparable with Friedman D et al $2013^{[14]}$ who studied 126 cases having SLN metastasis to predict the non-SLN status. He observed that $18.3 \%$ cases showed only SLN positivity with non-SLNs free from metastasis.

In present study, no association between age of patient and SLN metastasis was present which was comparable to findings of Koyama Y et al 2013. ${ }^{[15]}$

In present study, as the tumour size increased, metastasis was observed in SLN. The present study was comparable with findings of Koyoma Y et al 2013 ${ }^{[15]}$, Gajdos C et al $1999^{[16]}$ and Viale G et al $1998^{[17]}$ wherein association between tumour size and SLN metastasis were seen.

In present study association between tumour location and SLN positivity was observed. 15 out of 24 tumours (25\%) in upper outer quadrant showed SLN positive for metastasis whereas 1 out of 6 tumours (1.7\%) in lower inner quadrant showed SLN positive for metastasis. The present study is comparable with findings of Fein D A et al 1997..$^{[18]}$

In present study 13 cases were diagnosed as grade III invasive ductal carcinoma out of which 11 cases showed SLN metastasis (84.6\%). The present study is comparable with findings of Rivadeneira D et al 2000 ${ }^{[19]}$ and Olivotto I A et al $1998^{[20]}$ wherein the risk of SLN positivity increases with increase in grade of invasive ductal carcinoma.

\section{Conclusion}

Intraoperative frozen section assessment of sentinel lymph node proved to be quick and accurate method to detect the metastasis in the SLN with a sensitivity of $90.3 \%$, specificity of $100 \%$, positive predictive value of $100 \%$, negative predictive value of $90.6 \%$, diagnostic accuracy of $95 \%$ and false negative rate of $13.3 \%$. The sensitivity can further be improved with refinement in techniques such as expert visualization of blue coloured node intraoperatively, immediate processing of SLN, proper sectioning, staining and microscopic screening of slides. The positivity of SLN for metastasis is dependent upon certain characteristics of patients such as tumour size, tumour location and grade of tumour and independent of patient's age. However, in $16.1 \%$ of cases only sentinel lymph nodes are positive and these patients might undergo unnecessary axillary lymph node dissection. Hence, we can conclude that intraoperative frozen section evaluation of sentinel lymph node (SLN) to predict the status of non-SLN can be recommended only in selected patients in early stage of disease.

\section{Acknowledgements}

My sincere gratitude to Dr. P.Lokare, Dr. V. Wasnik, Dr. Ujwala Dhewale for helping me with the statistical analysis. I am grateful to Mr. A. V. Deshmukh, Mr. Prashant Kalmegh and all the non teaching staff for their technical support and for being ready to help whenever needed. I am thankful to my parents and my brother whose unflinching support has been a pillar of strength and sustenance in every sense of these words. Lastly, I am specially thankful to all the study participants and their guardians for allowing me to conduct this study on them.

\section{Reference}

1. Arafah M. Correlation of hormone receptors with her $2 /$ neu protein expression and the histological grade in invasive breast cancers in a cohort of Saudi Arabia. TJPATH. 2010;26(3):209.

2. D'cruz A. Standard treatment guidelines oncology [Internet]. 2018 [cited 1 November 2015]. Available from: http:// clinicalestablishments.nic.in/WriteReadData/329.pdf.

3. Asthana S, Labani P, Labani S. A review on cancer incidence in India from 25 population-based cancer registries. J NTR Univ Health Sci. 2015;4:150.

4. Mansel R, Fallowfield L, Kissin M, Goyal A, Newcombe R, Dixon J et al. Randomized multicenter trial of sentinel node biopsy versus standard axillary treatment in operable breast cancer: the ALMANAC trial. JNCI. 2006;98:599-609.

5. Samphao S, Eremin J, El-Sheemy M, Eremin O. Management of the axilla in women with breast cancer: current clinical practice and a new selective targeted approach. Annals of surgical oncology. 2008;15(5):1282-96.

6. Larson D, Weinstein M, Goldberg I, Silver B, Recht A, Cady B et al. Edema of the arm as a function of the extent of axillary surgery in patients with stage I-II carcinoma of the breast treated with primary radiotherapy. Int J Radiat Oncol Biol Phys. 1986;12(9):1575-82. 
7. Ivens D, Hoe AL, Podd TJ, Hamilton CR, Taylor I, Royle GT. Assessment of morbidity from complete axillary dissection. Br J Cancer. 1992;66(1):136-8.

8. Keramopoulos A, Tsionou C, Minaretzis D, Michalas S, Aravantinos D. Arm morbidity following treatment of breast cancer with total axillary dissection: a multivariated approach. Oncology. 1993;50(6):445-9.

9. Senkus E, Kyriakides S, Ohno S, Penault-Llorca F, Poortmans P, Rutgers E et al. Primary breast cancer: ESMO Clinical Practice Guidelines for diagnosis, treatment and follow-up. Ann Oncol. 2015;26 (5):v8-v30.

10. Lyman GH, Somerfield MR, Bosserman LD, Perkins CL, Weaver DL, Giuliano AE. Sentinel lymph node biopsy for patients with early-stage breast cancer: American Society for Clinical Oncology clinical practice guideline update. J Clin Oncol. 2017;35:561-4.

11. Mitchell ML. Frozen section diagnosis for axillary sentinel lymph nodes: the first six years. Mod Pathol. 2005;18:58-61.

12. Gipponi M, Bassetti C, Canavese G, Catturich A, Di Somma $\mathrm{C}$, Vecchio $\mathrm{C}$ et al. Sentinel lymph node as a new marker for therapeutic planning in breast cancer patients. J Surg Oncol. 2004;85(3):102-111.

13. Tille J, Egger J, Devillaz M, Vlastos G, Pelte F. Frozen section in axillary sentinel lymph nodes for diagnosis of breast cancer micrometastasis. Anticancer Res. 2009;29(11):4711-16.
14. Friedman D, Gipponi M, Murelli F, Meszaros P, Solari N, Massa $\mathrm{M}$ et al. Predictive factors of non-sentinel lymph node involvement in patients with invasive breast cancer and sentinel node micrometastases. Anticancer Res. 2013;33:4509-14.

15. Koyama Y, Ichikawa H, Sakata J, Sakata E, Tatsuda K, Hasegawa $\mathrm{M}$ et al. The association between sentinel lymph node metastasis and Ki-67 labeling index. Adv Breast Cancer Res. 2013;02:60-65.

16. Gajdos C, Tartter P, Bleiweiss I. Lymphatic invasion, tumor size, and age are independent predictors of axillary lymph node metastases in women with T1 breast cancers. Ann Surg. 1999;230(5):692-6.

17. Viale G, Zurrida S, Maiorano E, Mazzarol G, Pruneri G, Paganelli $G$ et al. Predicting the status of axillary sentinel lymph nodes in 4351 patients with invasive breast carcinoma treated in a single institution. Cancer. 2005;103:492-500.

18. Fein D, Fowble B, Hanlon A, Hooks M, Hoffman J, Sigurdson $\mathrm{E}$ et al. Identification of women with T1-T2 breast cancer at low risk of positive axillary nodes. J Surg Oncol. 1997;65:34-39.

19. Rivadeneira D, Simmons R, Christos P, Hanna K, Daly J, Osborne M. Predictive factors associated with axillary lymph node metastases in T1a and T1b breast carcinomas: analysis in more than 900 patients. J Am Coll of Surg. 2000;191:1-6.

20. Olivotto I, Jackson J, Mates D, Andersen S, Davidson W, Bryce $\mathrm{C}$ et al. Prediction of axillary lymph node involvement of women with invasive breast carcinoma: a multivariate analysis. Cancer. 1998;83:948-955.

*Corresponding author:

Dr. Aishwarya Vinod Bhongade Assistant Professor, Mailing Address: "SMIT", Swastik Chowk, Avadhutwadi, Godhni Road, Yavatmal-445001

Maharashtra, India

Phone: +919689246611

Email: aishwaryabhongade@gmail.com

Financial or other Competing Interests: None. 